\title{
Transductive Conformal Predictors
}

\author{
Vladimir Vovk \\ Department of Computer Science, \\ Royal Holloway, University of London, \\ Egham, Surrey, UK \\ v.vovk@rhul.ac.uk \\ http://vovk.net
}

\begin{abstract}
This paper discusses a transductive version of conformal predictors. This version is computationally inefficient for big test sets, but it turns out that apparently crude "Bonferroni predictors" are about as good in their information efficiency and vastly superior in computational efficiency.
\end{abstract}

Keywords: Conformal prediction, transduction, Bonferroni adjustment.

\section{Introduction}

The most standard learning problems are inductive: given a training set of labelled objects, the task is to come up with a predictor with a reasonable performance on unknown test objects. In typical transductive problems (7], Chapter VI, Sections 10-13, 6], Chapter 8) we are given both a training set of labelled objects and a test set of unlabelled objects; the task is to come up with a predictor, which may depend on both sets, with a reasonable performance on the test set. Conformal predictors (9, Chapter 2) are not transductive in this sense, although they do have a transductive flavour (see, e.g., 9], pp. 6-7).

The goal of this paper is to introduce a fully transductive version of conformal predictors. The basic definitions are given in Section 2, Section 3 introduces Bonferroni predictors, a simple and computationally efficient modification of conformal predictors adapted to the transductive framework. Sections 4 and 5 contain simple theoretical results about transductive conformal predictors and Bonferroni predictors. Section 6 reports on experimental results. Finally, Section 7 concludes.

The expression "transductive conformal predictors" has been used before (see, e.g., [3]) to refer to what is called "conformal predictors" in [9] and this paper. This agrees with our terminology, since conformal predictors are a special case of our transductive conformal predictors corresponding to a test set of size 1 .

\section{Transductive Conformal Predictors}

Let $z_{1}=\left(x_{1}, y_{1}\right), \ldots, z_{l}=\left(x_{l}, y_{l}\right)$ be a training set and $x_{l+1}, \ldots, x_{l+k}$ be a test set. The test set consists of objects $x_{j} \in \mathbf{X}$ and the training set consists of 
labelled objects, or examples, $z_{i}=\left(x_{i}, y_{i}\right) \in \mathbf{Z}:=\mathbf{X} \times \mathbf{Y}$. The object space $\mathbf{X}$, label space $\mathbf{Y}$, and example space $\mathbf{Z}:=\mathbf{X} \times \mathbf{Y}$ are fixed throughout the paper; they are assumed to be measurable spaces.

Transductive conformal predictors are determined by their transductive nonconformity measures, which are defined as follows. A transductive nonconformity measure is a measurable function $A: \mathbf{Z}^{*} \times \mathbf{Z}^{*} \rightarrow \mathbb{R}$ such that $A\left(\zeta_{1}, \zeta_{2}\right)$ does not depend on the ordering of $\zeta_{1}$. (For the specific transductive nonconformity measures used in this paper $A\left(\zeta_{1}, \zeta_{2}\right)$ will not depend on the ordering of $\zeta_{2}$ either.) The intuition is that $A\left(\zeta_{1}, \zeta_{2}\right)$ (the transductive nonconformity score) measures the lack of conformity of the "test set" $\zeta_{2}$ to the "training set" $\zeta_{1}$.

The transductive conformal predictor (TCP) corresponding to $A$ finds the prediction region for the test set $x_{l+1}, \ldots, x_{l+k}$ at a significance level $\epsilon \in(0,1)$ as follows:

- For each possible set of labels $\left(v_{1}, \ldots, v_{k}\right) \in \mathbf{Y}^{k}$ :

- set $y_{j}:=v_{j-l}$ and $z_{j}:=\left(x_{j}, y_{j}\right)$ for $j=l+1, \ldots, l+k$;

- compute the transductive nonconformity scores

$$
\alpha_{S}:=A\left(z_{\{1, \ldots, l+k\} \backslash S}, z_{S}\right),
$$

where $S$ ranges over all $(l+k) ! / l$ ! ordered subsets $\left(s_{1}, \ldots, s_{k}\right)$ of $\{1, \ldots, l+k\}$ of size $k, z_{S}$ stands for the sequence $\left(z_{s_{1}}, \ldots, z_{s_{k}}\right)$ (when $\left.S=\left(s_{1}, \ldots, s_{k}\right)\right)$, and $z_{\{1, \ldots, l+k\} \backslash S}$ stands for $z_{B}, B$ being any ordering of $\{1, \ldots, l+k\} \backslash S^{\prime}$ and $S^{\prime}$ being the set of all elements of $S$ (it does not matter which ordering is chosen, by the definition of a transductive nonconformity measure);

- compute the p-value

$$
p\left(v_{1}, \ldots, v_{k}\right):=\frac{\left|\left\{S \mid \alpha_{S} \geq \alpha_{(l+1, \ldots, l+k)}\right\}\right|}{(l+k) ! / k !},
$$

where $S$ ranges, as before, over all $(l+k) ! / l$ ! ordered subsets of $\{1, \ldots, l+$ $k$ \} of size $k$, and $|\ldots|$ stands for the size of a set.

- Output the prediction region

$$
\Gamma^{\epsilon}\left(z_{1}, \ldots, z_{l}, x_{l+1}, \ldots, x_{l+k}\right):=\left\{\left(v_{1}, \ldots, v_{k}\right) \in \mathbf{Y}^{k} \mid p\left(v_{1}, \ldots, v_{k}\right)>\epsilon\right\} .
$$

Smoothed TCPs are defined in the same way except that (11) is replaced by

$$
p\left(v_{1}, \ldots, v_{k}\right):=\frac{\left|\left\{S \mid \alpha_{S}>\alpha_{(l+1, \ldots, l+k)}\right\}\right|+\theta\left|\left\{S \mid \alpha_{S}=\alpha_{(l+1, \ldots, l+k)}\right\}\right|}{(l+k) ! / k !}
$$

where $\theta$ are random variables distributed uniformly on $[0,1]$ (no independence between different sets of postulated labels $v_{1}, \ldots, v_{k}$ is required, but later on when we consider the online prediction protocol we will assume that $\theta$ are independent between different trials). 
A nonconformity measure can now be defined as the restriction of a transductive nonconformity measure to the domain $\mathbf{Z}^{*} \times \mathbf{Z}$ (we identify a 1-element sequence with its only element). Nonconformity measures are well studied and there are many useful examples of them (see, e.g., [9]).

An interesting class of transductive nonconformity measures can be obtained from nonconformity measures. Let $\mathbb{R}$ be the set of real numbers. A simple nonconformity aggregator is a function $M: \mathbb{R}^{*} \rightarrow \mathbb{R}$ that is symmetric and increasing in each argument. (The requirement that $M$ be symmetric, i.e., $M(\zeta)$ not depend on the ordering of $\zeta$, is not necessary but convenient for the following discussion. The requirement that $M$ be increasing in each argument is not necessary either but very natural.) With each nonconformity measure $A$ and simple nonconformity aggregator $M$ we can associate the transductive nonconformity measure

$$
A_{M}\left(\left(z_{1}, \ldots, z_{l}\right),\left(z_{l+1}, \ldots, z_{l+k}\right)\right):=M\left(\alpha_{l+1}, \ldots, \alpha_{l+k}\right)
$$

where

$$
\begin{array}{r}
\alpha_{j}:=A\left(\left(z_{1}, \ldots, z_{l}, z_{l+1}, \ldots, z_{j-1}, z_{j+1}, \ldots, z_{l+k}\right), z_{j}\right) \\
\quad j=l+1, \ldots, l+k .
\end{array}
$$

Our experiments in Section [6] use the Nearest Neighbour nonconformity measure

$$
A\left(\left(\left(x_{1}, y_{1}\right), \ldots,\left(x_{l}, y_{l}\right)\right),(x, y)\right):=\frac{\min _{i=1, \ldots, l: y_{i}=y} d\left(x, x_{i}\right)}{\min _{i=1, \ldots, l: y_{i} \neq y} d\left(x, x_{i}\right)},
$$

where $d$ is a distance, and the max nonconformity aggregator

$$
M\left(\alpha_{1}, \ldots, \alpha_{k}\right):=\max \left(\alpha_{1}, \ldots, \alpha_{k}\right) .
$$

Remark 1. Alternatively, we could set $\alpha_{j}:=A\left(\left(z_{1}, \ldots, z_{l}\right), z_{j}\right)$ in (3) (cf. (12) below), but this would adversely affect the already low computational efficiency of TCPs in our experiments in Section 6.

\section{Rank-Based Transductive Conformal Predictors}

The notion of a simple nonconformity aggregator can be generalized as follows. A nonconformity aggregator is a function $M: \mathbb{R}^{*} \times \mathbb{R}^{*} \rightarrow \mathbb{R}$ such that $M\left(\zeta_{1}, \zeta_{2}\right)$ depends neither on the ordering of $\zeta_{1}$ nor on the ordering on $\zeta_{2}$. (The most natural class of nonconformity aggregators is where $M\left(\zeta_{1}, \zeta_{2}\right)$ is decreasing in every element of $\zeta_{1}$ and increasing in every element of $\zeta_{2}$, but it is too narrow for our purposes.) With each nonconformity measure $A$ and nonconformity aggregator $M$ we associate the transductive nonconformity measure

$$
A_{M}\left(\left(z_{1}, \ldots, z_{l}\right),\left(z_{l+1}, \ldots, z_{l+k}\right)\right):=M\left(\left(\alpha_{1}, \ldots, \alpha_{l}\right),\left(\alpha_{l+1}, \ldots, \alpha_{l+k}\right)\right)
$$

where

$$
\alpha_{i}:=A\left(\left(z_{1}, \ldots, z_{i-1}, z_{i+1}, \ldots, z_{l}, z_{l+1}, \ldots, z_{l+k}\right), z_{i}\right), \quad i=1, \ldots, l,
$$


and $\alpha_{l+1}, \ldots, \alpha_{l+k}$ are defined by (3). We identify each simple nonconformity aggregator $M$ with the nonconformity aggregator

$$
M^{\dagger}\left(\left(\alpha_{1}, \ldots, \alpha_{l}\right),\left(\alpha_{l+1}, \ldots, \alpha_{l+k}\right)\right):=M\left(\alpha_{l+1}, \ldots, \alpha_{l+k}\right) .
$$

For transductive nonconformity measures obtained from nonconformity measures and nonconformity aggregators, the p-value (1) as function of the nonconformity scores $\alpha_{i}$ of individual examples reduces to the well-known notion of a one-sided permutation test (see, e.g., 11, Section 1.7.E). In classical nonparametric statistics, the most popular permutation tests are rank tests, and we will give corresponding definitions in our current context. Let $\mathbb{N}:=\{1,2, \ldots\}$. A (simple) rank aggregator is a function $M: \mathbb{N}^{*} \rightarrow \mathbb{N}$ that is symmetric and increasing in each argument. The corresponding nonconformity aggregator is

$$
M^{\prime}\left(\left(\alpha_{1}, \ldots, \alpha_{l}\right),\left(\alpha_{l+1}, \ldots, \alpha_{l+k}\right)\right):=M\left(R_{l+1}, \ldots, R_{l+k}\right),
$$

where $R_{1}, \ldots, R_{l+k}$ are the ranks of $\alpha_{1}, \ldots, \alpha_{l+k}$, respectively, in the multiset $\left\{\alpha_{1}, \ldots, \alpha_{l+k}\right\}$. Formally, $R_{i}$ is defined as

$$
R_{i}:=\left|\left\{j=1, \ldots, l+k \mid \alpha_{j}<\alpha_{i}\right\}\right|+1 .
$$

If there are no ties (i.e., equal elements in $\left\{\alpha_{1}, \ldots, \alpha_{l+k} \int\right)$, this is the usual notion of a rank; in the presence of ties, our definition is somewhat non-standard giving each tie the smallest of the ranks that it spans. (And this definition causes a counterintuitive behaviour of the definition (7), where $M^{\prime}$ is not necessarily increasing in $\alpha_{j}, j \in\{l+1, \ldots, l+k\}$, even in the case where $M$ is the $\max$ nonconformity aggregator (5).)

The most popular rank aggregator in classical nonparametric statistics is the ranksum aggregator

$$
M\left(R_{1}, \ldots, R_{k}\right):=R_{1}+\cdots+R_{k}
$$

which is used in the Wilcoxon ranksum test (see [11] or [1, Section 1.2). Using the ranksum aggregator, however, produces very poor results (see Appendix B of [10] and the right panel of Figure 4) when the efficiency of TCPs is measured by the number of multiple predictions that they produce, as in this paper (see Section 6 below).

Notice that the nonconformity aggregator (5) is equivalent (in the sense of leading to the same TCP) to the rank aggregator $M^{\prime}\left(R_{1}, \ldots, R_{k}\right):=$ $\max \left(R_{1}, \ldots, R_{k}\right)$. The corresponding TCP will be called the rankmax TCP (and the TCP corresponding to (8) will be called the ranksum TCP).

It is easy to give an explicit representation of the rankmax TCP. Remember that the size of the training set is $l$ and the size of the test set is $k$ and suppose that the value of the rankmax test statistic $\max \left(R_{l+1}, \ldots, R_{l+k}\right)$ is $t$. The probability that a random subset $\left\{s_{1}, \ldots, s_{k}\right\}$ of $\{1, \ldots, l+k\}$ of size $k$ will lead to a value of the test statistic $\max \left(R_{s_{1}}, \ldots, R_{s_{k}}\right)$ of at least $t$ can be found as 1 minus the probability that a random subset of $\{1, \ldots, l+k\}$ of size $k$ is covered 
by a fixed subset of $\{1, \ldots, l+k\}$ of size $t-1$ (namely, by the set of indices $i$ with $\left.R_{i}<t\right)$. In other words, the p-value is

$$
1-\frac{\left(\begin{array}{c}
t-1 \\
k
\end{array}\right)}{\left(\begin{array}{c}
l+k \\
k
\end{array}\right)}=1-\frac{(t-1) ! l !}{(t-1-k) !(l+k) !}
$$

(which is understood to be 1 when $t \leq k$ ).

\section{Bonferroni Predictors}

Unfortunately, transductive conformal predictors are computationally inefficient, especially if we want to predict many test objects at once: we have to go over all $|\mathbf{Y}|^{k}$ combinations of labels for the test set. (Even if $A\left(\zeta_{1}, \zeta_{2}\right)$ does not depend on the ordering of $\zeta_{2}$, there are no computational savings unless the test set contains many identical objects.) We next introduce a family of region predictors based on the idea of the Bonferroni adjustment of p-values. In brief, a Bonferroni predictor computes a p-value for each test object separately and then combines the $k \mathrm{p}$-values into one p-value using the Bonferroni formula

$$
p:=\min \left(k p_{1}, \ldots, k p_{k}, 1\right) .
$$

The full description of the Bonferroni predictor $(B P)$ corresponding to a nonconformity measure $A$ is as follows:

- For each object $x_{j}, j \in\{l+1, \ldots, l+k\}$, in the test set and each possible label $v \in \mathbf{Y}$ :

- set $y_{j}:=v$ and $z_{j}:=\left(x_{j}, y_{j}\right)$;

- compute the nonconformity scores

$$
\begin{aligned}
& \alpha_{i}:=A\left(\left(z_{1}, \ldots, z_{i-1}, z_{i+1}, \ldots, z_{l}, z_{j}\right), z_{i}\right), \quad i=1, \ldots, l, \\
& \alpha_{j}:=A\left(\left(z_{1}, \ldots, z_{l}\right), z_{j}\right)
\end{aligned}
$$

- compute the p-value

$$
p_{j-l}(v):=\frac{\left|\left\{i=1, \ldots, l \mid \alpha_{i} \geq \alpha_{j}\right\}\right|+1}{l+1} .
$$

- Output the prediction region

$$
\Gamma^{\epsilon}\left(z_{1}, \ldots, z_{l}, x_{l+1}, \ldots, x_{l+k}\right):=\prod_{j=l+1}^{l+k}\left\{v \mid p_{j-l}(v)>\epsilon / k\right\},
$$

where $\epsilon \in(0,1)$ is the significance level.

Notice that the prediction region (14) output by the BP can be rewritten in the form (2) if we define

$$
p\left(v_{1}, \ldots, v_{k}\right):=\min \left(k p_{1}\left(v_{1}\right), \ldots, k p_{k}\left(v_{k}\right), 1\right)
$$

(cf. (10) ). 
It is difficult to compare the rankmax TCP and the corresponding BP theoretically, but the following intermediate notion facilitates a comparison. The semi-Bonferroni predictor $(S B P)$ is defined as follows:

- For each possible set of labels $\left(v_{1}, \ldots, v_{k}\right) \in \mathbf{Y}^{k}$ for the test set:

- set $y_{j}:=v_{j-l}$ and $z_{j}:=\left(x_{j}, y_{j}\right)$ for $j=l+1, \ldots, l+k$;

- compute nonconformity scores $\alpha_{i}, i=1, \ldots, l+k$, by

$$
\alpha_{i}:=A\left(\left(z_{1}, \ldots, z_{i-1}, z_{i+1}, \ldots, z_{l+k}\right), z_{i}\right), \quad i=1, \ldots, l+k
$$

(cf. (6) and (3); the main difference of (16) from (6) and (3) is that (16) involves the true training examples and test objects whereas (6) and (3) involve arbitrary subsets of size $l$ and $k$ of the union of the training set and the test set with postulated labels);

- compute the p-value (13) for each $j=l+1, \ldots, l+k$ and merge these p-values using (15).

- Output the prediction region (2).

Notice that the SBP becomes identical to the BP when (16) is replaced by (11) and (12) for $j=l+1, \ldots, l+k$.

The following lemma shows that SBPs are usually weaker than the corresponding rankmax TCPs. (However, in Remark 2 and Section 6 we will see that the difference can be surprisingly small.)

Lemma 1. Suppose all nonconformity scores (16) are different. The p-value (9) produced by a rankmax TCP does not exceed the p-value (15) produced by the corresponding SBP.

Proof. Let $t$ be the value of the rankmax test statistic, as defined at the end of Section 2. We are required to prove

$$
1-\frac{\left(\begin{array}{c}
t-1 \\
k
\end{array}\right)}{\left(\begin{array}{c}
l+k \\
k
\end{array}\right)} \leq k \frac{l+k-t+1}{l+1} .
$$

Indeed, the left-hand side of (17) is identical to (9), and the ratio on the righthand side of (17) is the smallest of the p-values (13) over $j$ (cf. (15)). The statement that the ratio on the right-hand side of (17) is the smallest of the pvalues (13) depends on (16) being all different (in fact, it is sufficient to assume that the maximum in the definition of the rankmax test statistic $t$ is attained on only one test object). Notice, however, that the right-hand side of (17) is always an upper bound on the SBP p-value; this fact will be used in our discussions below.

We will prove a slightly stronger inequality that (17) replacing the denominator $l+1$ by $l+k$. In principle, $t$ can take any value in $\{1, \ldots, l+k\}$, but we can assume, without loss of generality, that $t \in\{k+1, \ldots, l+k\}$ : if $t \leq k$, the 
left-hand side of (17) is 1 by definition and the right-hand side is at least 1 (even when $l+1$ is replaced by $l+k$ ). Rewriting (17) (with $l+k$ in place of $l+1$ ) as

$$
1-\frac{(t-1)(t-2) \cdots(t-k)}{k !\left(\begin{array}{c}
l+k \\
k
\end{array}\right)} \leq k \frac{l+k-t+1}{l+k},
$$

we can assume that $t \in[k+1, l+k]$. Since the fraction on the left-hand side of (18) is a convex function of $t$ (the second derivative is obviously nonnegative) and for $t:=k+l$ (18) holds (it becomes $k /(l+k) \leq k /(l+k)$ ), it suffices to prove that the derivative in $t$ of the left-hand side of (18) at the point $l+k$ is equal to or exceeds the derivative of the right-hand side:

$$
-\frac{(\Gamma(t) / \Gamma(t-k))_{t=l+k}^{\prime}}{k !\left(\begin{array}{c}
l+k \\
k
\end{array}\right)} \geq k \frac{-1}{l+k},
$$

where $\Gamma$ is the gamma function, $\Gamma(n)=(n-1)$ ! for $n \in \mathbb{N}$. By the definition of the digamma function $\psi$, the last inequality can be rewritten as

$$
\frac{\Gamma(l+k)}{\Gamma(l)}(\psi(l+k)-\psi(l)) \leq \frac{k}{l+k} k !\left(\begin{array}{c}
l+k \\
k
\end{array}\right),
$$

which simplifies to

$$
\psi(l+k)-\psi(l) \leq \frac{k}{l}
$$

The well-known expression for $\psi$ at the integer values of its argument (see, e.g., http://dlmf.nist.gov/5.4.14) allows us to rewrite the last inequality as

$$
\frac{1}{l}+\frac{1}{l+1}+\cdots+\frac{1}{l+k-1} \leq \frac{k}{l},
$$

which is obviously true.

Remark 2. The proof of Lemma 1] shows that the inequality (17) is strict whenever $k>1$ (for $k=1$ the two p-values coincide). Three factors contribute to its being strict: the SBP p-value is larger than the rankmax TCP p-value at $t=l+k$; as function of $t$, the SBP p-value has a steeper (negative) slope at $t=l+k$; besides, to the left of $t=l+k$ the SBP p-value goes in a straight line whereas the rankmax TCP p-value veers down. This is illustrated in Figure 1 for $l=1000$ and $k=2$ (typical values for our experiments reported in Section [6); the first two factors, however, are not noticeable.

It is plausible that a BP usually produces somewhat smaller p-values (and, therefore, somewhat smaller prediction regions) than the corresponding SBP: the only difference is that, when computing p-values, the SBP uses more test objects with arbitrarily assigned labels, and this may lead to a greater distortion of the nonconformity scores. 

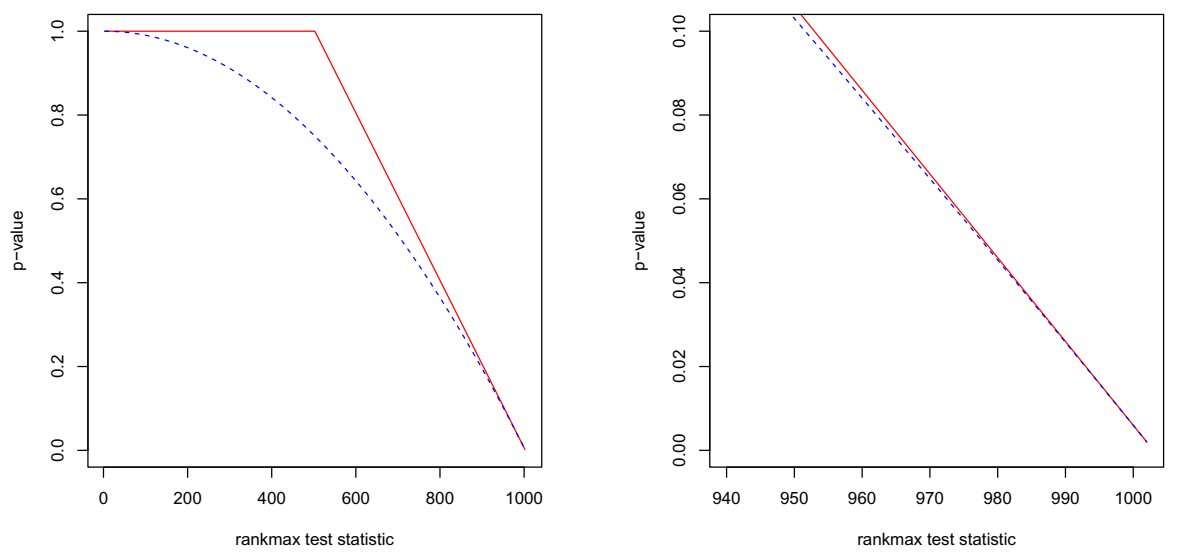

Fig. 1. Left panel: SBP p-values (the solid red line) and rankmax TCP p-values (the dashed blue line) for $l=1000$ and $k=2$ as functions of $t$. Right panel: the lower right corner of the left panel.

\section{Validity}

The strongest notion of validity for conformal and related predictors can be stated in the online mode. Suppose we are given a sequence of positive integer numbers $k_{1}, k_{2}, \ldots$ and the incoming sequence of examples is $z_{1}=\left(x_{1}, y_{1}\right), z_{2}=$ $\left(x_{2}, y_{2}\right), \ldots$; set $l_{n}:=\sum_{i=1}^{n-1} k_{i}$ (in particular, $l_{1}:=0$ ). At trial $n=1,2, \ldots$ of the online prediction protocol, Predictor predicts the $k_{n}$ labels $y_{l_{n}+1}, \ldots, y_{l_{n}+k_{n}}$ given the $l_{n}$ examples $z_{1}, \ldots, z_{l_{n}}$ and $k_{n}$ objects $x_{l_{n}+1}, \ldots, x_{l_{n}+k_{n}}$. The prediction is a subset $\Gamma_{n}$ of $\mathbf{Y}^{k_{n}}$. It can be multiple $\left(\left|\Gamma_{n}\right|>1\right)$, singleton $\left(\left|\Gamma_{n}\right|=1\right)$, or empty $\left(\left|\Gamma_{n}\right|=0\right)$. Predictor makes an error if $\left(y_{l_{n}+1}, \ldots, y_{l_{n}+k_{n}}\right) \notin \Gamma_{n}$.

In this section we assume either that the sequence of examples $z_{1}, z_{2}, \ldots$ is infinite and the examples are produced independently from the same probability distribution on $\mathbf{Z}$, or that the sequence of examples is finite, $z_{1}, \ldots, z_{N}$, and produced from an exchangeable probability distribution on $\mathbf{Z}^{N}$.

The following simple result states the validity of TCPs in the online mode; its proof is standard (see, e.g., 8] or [9], Section 8.7) and is omitted.

Theorem 1. In the online mode, a smoothed TCP makes errors with probability $\epsilon$ (the significance level) independently at different trials.

A suitable version of validity in the absence of smoothing is conservative validity, i.e., being dominated by a sequence of independent Bernoulli trials with probability of success equal to the significance level; for details, see [9], p. 21. By Theorem 11. TCPs are conservatively valid:

Corollary 1. In the online mode, each TCP is conservatively valid. 
Proof. Each TCP is conservatively valid since it can only make an error when the corresponding smoothed TCP (i.e., the smoothed TCP based on the same transductive nonconformity measure) makes an error.

Lemma 1 suggests that SBPs can be regarded as conservatively valid for practical purposes, since an SBP can make an error only when the corresponding rankmax TCP makes an error, unless there are ties among nonconformity scores. (However, in general, it is not always true that an SBP can make an error only when the corresponding rankmax TCP makes an error. Consider, e.g., the case where $k=2$ and the nonconformity scores of the two test examples are equal and exceed the nonconformity scores of all training examples; in this case, the SBP p-value will be smaller than the rankmax TCP p-value unless $l=1$.)

Theorem 2. In the online mode, each BP is conservatively valid.

Proof (sketch). The proof follows the scheme of the proof in Appendix A.1 of 8$]$. Given the bag $\left\{z_{1}, \ldots, z_{l_{n}+k_{n}} \int\right.$ and under the assumption of exchangeability, the probability that the $\mathrm{BP}$ will make an error at trial $n$ for a given test example (e.g., for the second example in the test set) is at most $\epsilon / k_{n}$. Therefore, the probability that it will make an error for some of the $k_{n}$ test examples is at most $\epsilon$. We can increase the indicator function of making an error to obtain a Bernoulli random variable with probability of success equal to $\epsilon$ (this might involve extending the probability space). It remains to notice that whether an error is made at trial $n$ is determined by the bag $\left\{z_{1}, \ldots, z_{l_{n}} \int\right.$ and examples $z_{l_{n}+1}, \ldots, z_{l_{n}+k_{n}}$ (cf. Lemma 2 in [8]).

\section{$5 \quad$ Universality}

A transductive confidence predictor is a measurable strategy for Predictor in the online prediction protocol (as described in the previous section) depending on a parameter $\epsilon \in(0,1)$ (the significance level) in such a way that for each training set and each test set the prediction at a larger significance level is a subset of the prediction at a smaller significance level. We say that the transductive confidence predictor is conservatively valid if the sequence of errors that it makes at any significance level $\epsilon$ is dominated by a sequence of independent Bernoulli trials with probability of success $\epsilon$. We say that it is invariant if, when fed with examples $z_{1}, \ldots, z_{l_{n}}$ and objects $x_{l_{n}+1}, \ldots, x_{l_{n}+k_{n}}$ at any trial $n$, it issues the same prediction regardless of the ordering of $z_{1}, \ldots, z_{l_{n}}$. And we say that a transductive confidence predictor $\Gamma^{\prime}$ is at least as good as another transductive confidence predictor $\Gamma^{\prime \prime}$ if at any significance level $\epsilon$ the prediction region issued by $\Gamma^{\prime}$ is completely covered by the prediction region issued by $\Gamma^{\prime \prime}$. The following result, whose proof is omitted in this version of the paper, can be proved similarly to Theorem 2.6 in 9 .

Theorem 3. Suppose $\mathbf{Z}$ is a Borel space. For any conservatively valid transductive confidence predictor $\Gamma$ there exists a transductive conformal predictor $\Gamma^{\prime}$ that is at least as good as $\Gamma$. 

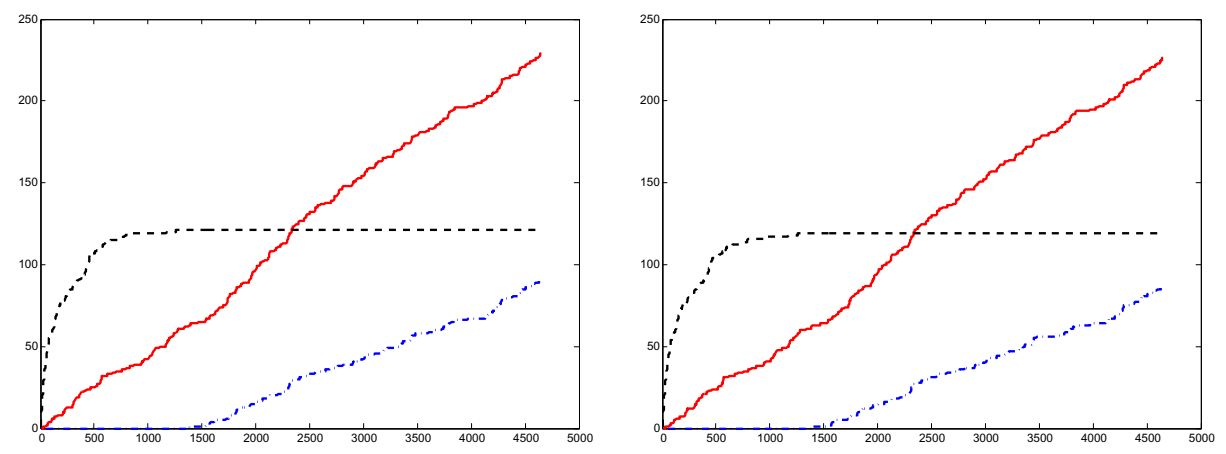

Fig. 2. Left panel: the performance of the rankmax TCP based on Nearest Neighbour for tangent distance on the USPS data set (randomly permuted) for the size $k=2$ of test sets and significance level $5 \%$. The cumulative errors are shown with a solid red line, multiple predictions with a dashed black line, and empty predictions with a dash-dot blue line. Right panel: the analogous picture for the BP.

Theorem 3 says that TCPs are universal in the sense of dominating all conservatively valid transductive confidence predictors. In particular, for any BP there is a TCP that is at least as good as that BP. However, in the next section we will see that the rankmax TCP corresponding to the same nonconformity measure does not always satisfy this property.

\section{Experiments}

In our experiments we will use the standard USPS data set of hand-written digits. The training set (7291 examples) is merged with the test set (2007 examples) and the resulting data set of 9298 examples is randomly permuted, to make sure the assumption of exchangeability is satisfied. The prediction protocol is online. In a typical scenario the digits might arrive in batches of $k=5$ digits and represent American zip codes (in this case, however, the exchangeability assumption is only a crude approximation, since the digits within the same zip code are likely to be written by the same person). However, the TCP and SBP are too computationally inefficient to be applied in this case, and for comparing them with the $\mathrm{BP}$ we first consider online prediction of batches of $k=2$ digits; intuitively, our task is to recognize a two-digit number.

We always use the Nearest Neighbour nonconformity measure (4), where $d$ is tangent distance [5], and study empirically the corresponding rankmax TCP, SBP, and BP. As the significance level we always take $5 \%$. The left panel of Figure 2 shows the performance of the rankmax TCP using three functions: the cumulative number of errors made over the trials $1, \ldots, n$ as function of $n$, the cumulative number of multiple predictions made over the trials $1, \ldots, n$ as function of $n$, and the cumulative number of empty predictions over the trials $1, \ldots, n$ as function of $n$. The performance of the SBP and BP as measured 

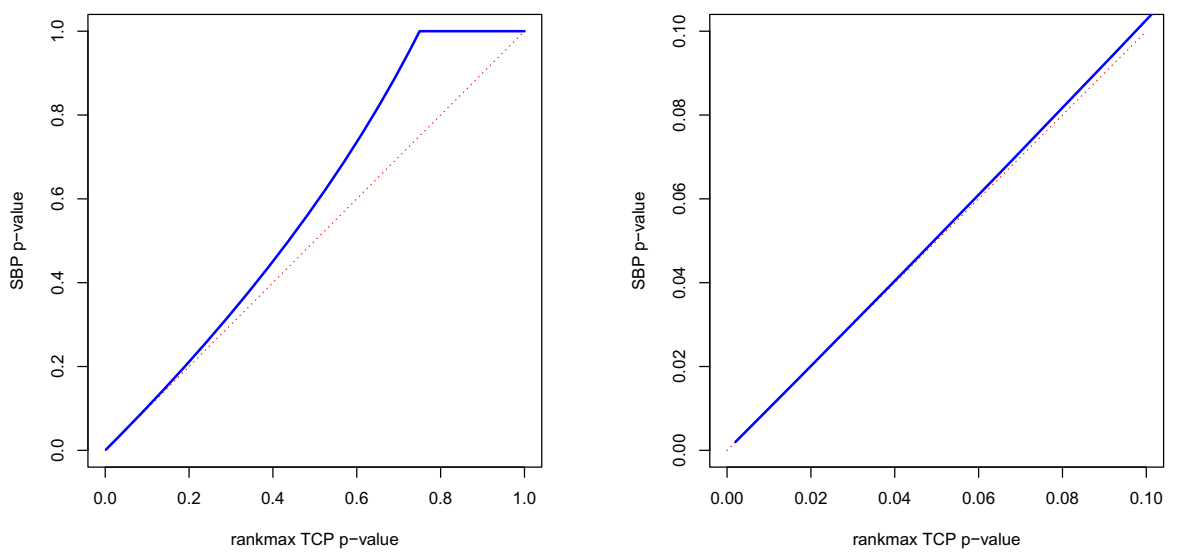

Fig. 3. Left panel: the p-values produced by an SBP vs the p-values produced by the corresponding rankmax TCP (the solid blue line) for the size $l=1000$ of the training set and $k=2$ of the test set. Right panel: the lower left corner of the left panel.

by these functions is very similar; only the latter is shown in the right panel of Figure 2, but all three graphs are visually indistinguishable. The BP even makes 2 fewer multiple predictions than the rankmax TCP, which confirms the claim made in Section 5 that the rankmax TCP corresponding to the same nonconformity measure as a given $\mathrm{BP}$ is not always at least as good as that BP. (It is not true in general that the BP always makes fewer multiple predictions than the corresponding rankmax TCP. It just happens to be true for tangent distance and seed 0 for the MATLAB pseudorandom number generator; e.g., the BP makes slightly more multiple predictions for Euclidean distance and seed 0.) The SBP makes one more multiple prediction than the rankmax TCP, which agrees with Lemma 1.

The cause of the similarity between the plots of the type shown in Figure 2 for the rankmax TCP and corresponding SBP is illustrated by Figure 3 (essentially a version of Figure 11, which shows the p-values produced by the SBP plotted against the respective p-values produced by the corresponding rankmax TCP, assuming there are no ties among the nonconformity scores. When the p-values are small, they are remarkably close to each other. And even without making any assumptions, we can still see that the SBP p-values are never significantly worse than the respective rankmax TCP p-values, assuming the latter are not too large.

The main advantage of BPs is that they are much more computationally efficient than both TCPs and SBPs. Because of their computational efficiency, it is very easy to produce the analogue of the right panel of Figure 2 for $k=5$ (as in American zip codes): see the left panel of Figure 4 but it is not clear at all how to make the computations for rankmax TCPs and SBPs feasible, even for moderately large $k$. 

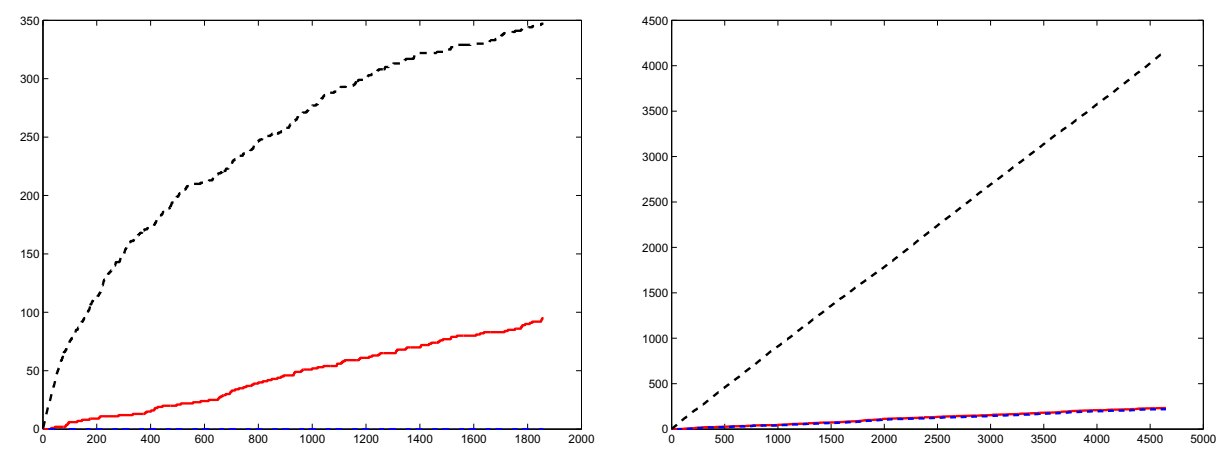

Fig. 4. Left panel: the performance of the $\mathrm{BP}$ for the size $k=5$ of test sets. Right panel: the performance of the ranksum TCP for $k=2$ (very poor). The setting is as in Figure 2 the prediction algorithms are based on Nearest Neighbour and tangent distance; the cumulative errors are shown with a solid red line, multiple predictions with a dashed black line, and empty predictions with a dash-dot blue line; the significance level is $5 \%$.

\section{Conclusion}

Based on our theoretical and empirical results, the preliminary recommendation is to use Bonferroni predictors in transductive problems: as compared to rankmax TCPs and SBPs, they enjoy the same theoretical validity guarantees, have comparable predictive performance empirically, but are much more computationally efficient.

The conclusion is preliminary since our empirical comparison in Section 6 only covers TCPs for a small size $k$ of the test set, namely $k=2$. The computational inefficiency of TCPs greatly complicates their empirical comparison with the BPs and SBPs for large values of $k$.

The comparison is much more straightforward in the case of transductive and Bonferroni extensions of inductive conformal predictors ([4]; 9], Section 4.1), and it can be shown that the two extensions produce similar p-values in practically important cases: see [10], Appendix A, for details.

Acknowledgments. I am grateful to Harris Papadopoulos for a discussion at COPA 2012 that rekindled my interest in transduction. Thanks to Wouter Koolen for illuminating discussions and for writing a MATLAB program for the Wilcoxon ranksum test (the standard programs in MATLAB and $R$ produce unsatisfactory results in the default mode and are prohibitively slow in the exact mode). My thanks also go to the COPA 2013 reviewers, whose comments have helped me in improving the presentation and suggested new directions of research. In my experiments I used the $\mathrm{C}$ program for computing tangent distance written by Daniel Keysers and adapted to MATLAB by Aditi Krishn. 
This work was partially supported by the Cyprus Research Promotion Foundation (research contract TPE/ORIZO/0609(BIE)/24) and by EPSRC (grant $\mathrm{EP} / \mathrm{K} 033344 / 1)$.

\section{References}

1. Lehmann, E.L.: Nonparametrics: Statistical Methods Based on Ranks, revised 1st edn. Springer, New York (2006)

2. National Institute of Standards and Technology: Digital library of mathematical functions (October 1, 2012), http://dlmf.nist.gov/

3. Nouretdinov, I., Costafreda, S.G., Gammerman, A., Chervonenkis, A., Vovk, V., Vapnik, V., Fu, C.H.Y.: Machine learning classification with confidence: Application of transductive conformal predictors to MRI-based diagnostic and prognostic markers in depression. Neuroimage 56, 809-813 (2011)

4. Papadopoulos, H., Vovk, V., Gammerman, A.: Qualified predictions for large data sets in the case of pattern recognition. In: Proceedings of the First International Conference on Machine Learning and Applications, pp. 159-163. CSREA Press, Las Vegas (2002)

5. Simard, P., LeCun, Y., Denker, J.: Efficient pattern recognition using a new transformation distance. In: Hanson, S., Cowan, J., Giles, C. (eds.) Advances in Neural Information Processing Systems, vol. 5, pp. 50-58. Morgan Kaufmann, San Mateo (1993)

6. Vapnik, V.N.: Statistical Learning Theory. Wiley, New York (1998)

7. Vapnik, V.N., Chervonenkis, A.Y.: Theory of Pattern Recognition. Nauka, Moscow (1974) (in Russian); German translation:Theorie der Zeichenerkennung, Akademie, Berlin (1979)

8. Vovk, V.: On-line Confidence Machines are well-calibrated. In: Proceedings of the Forty Third Annual Symposium on Foundations of Computer Science, pp. 187-196. IEEE Computer Society, Los Alamitos (2002)

9. Vovk, V., Gammerman, A., Shafer, G.: Algorithmic Learning in a Random World. Springer, New York (2005)

10. Vovk, V.: Transductive conformal predictors. On-line Compression Modelling project (New Series), Working Paper 8, an extended version of this paper (2013), http://alrw.net

11. Wilcoxon, F.: Individual comparisons by ranking methods. Biometrics Bulletin 1, 80-83 (1945) 